\title{
Indirect constraints on the dark matter interpretation of excess positrons seen by AMS-02
}

\author{
Man Ho Chan \\ Department of Science and Environmental Studies, \\ The Hong Kong Institute of Education \\ Tai Po, New Territories, Hong Kong, China
}

\begin{abstract}
Recently, an excess of high energy positrons in our Galaxy has been observed by AMS-02. The spectrum obtained can be best fitted with the annihilation of $\sim \mathrm{TeV}$ dark matter particles. However, recent analysis of Dwarf galaxies by Fermi/LAT observations highly constrains the TeV dark matter annihilation cross-section, and rules out the $b \bar{b}$ and all the leptophilic channels except $4-\mu$ channel. In this article, I show that the remaining possible $4-\mu$ channel is also ruled out by using the observational data from cool-core clusters. Therefore, all the leptophilic channels that can account for the excess positrons seen in AMS-02, HEAT, and PAMELA are ruled out.
\end{abstract}




\section{A. Introduction}

Recently, the observations of high-energy positrons by HEAT [1], PAMELA [2] and AMS02 [3 5] reveal some excess emissions in our Galaxy. These excess emissions cannot be easily explained by standard astrophysical mechanisms [6]. Some studies propose that pulsars could generate enough high-energy positrons to account for the excess [7-13]. On the other hand, many studies of the positron excess are now focusing on the annihilation of dark matter particles [14 19]. Later, Boudaud et al. (2015) perform a detailed analysis on the latest AMS-02 measurements and give a robust constraint on different annihilation channels such as $b \bar{b}, e^{+} e^{-}, W^{+} W^{-}$and $\mu^{+} \mu^{-}$. They use more accurate data and look at a set of 1623 different combinations of the cosmic ray transportation parameters. They find that the allowed dark matter parameter space increases, and some other channels such as $4-\mu$ and $4-\tau$ can now provide excellent fits to the positron excess. The ranges of the best-fit annihilation cross-section $\langle\sigma v\rangle$ and dark matter mass $m_{\chi}$ are $\langle\sigma v\rangle \sim\left(10^{-24}-10^{-21}\right)$ $\mathrm{cm}^{3} \mathrm{~s}^{-1}$ and $m_{\chi} \sim 0.1-10 \mathrm{TeV}$ respectively [20]. The annihilation cross-sections obtained are larger than the expected one for a thermal relic. Such an enhancement could arise due to substructures in the dark matter distribution [6] or Sommerfeld enhancement [21].

Based on the result from [20], Lopez et al. (2015) use the Fermi/LAT gamma-ray data from dwarf galaxies to further constrain the cross-section and dark matter mass, especially for the $b \bar{b}$ and leptophilic annihilation channels. They find that all except the $4-\mu$ channel are ruled out [6]. Moreover, the multichannel combinations into $b \bar{b}$ and leptons are also excluded unless the branching ratios are allowed to deviate from their best-fit values [6]. The $4-\mu$ channel can escape from Fermi/LAT constraints because the emission of gammaray from dark matter annihilation is much less than the other channels. Most of the energy is given to the positron-electron pairs.

If the proposed $\mathrm{TeV}$ dark matter is really the dark matter in our universe, we may also constrain the dark matter properties by using the observational data in galaxy clusters. In this article, I show that the luminosity due to the cooling of the electron-positron pairs produced from dark matter annihilation through $4-\mu$ channel is larger than the observed luminosity in some nearby cool-cored galaxy clusters. In other words, all the leptophilic channels that can account for the positron excess are ruled out. 


\section{B. Dark matter annihilation in galaxy clusters}

In the following, we consider dark matter annihilation through $4-\mu$ channel: $\chi \chi \rightarrow$ $\phi \phi \rightarrow 4 \mu$, where $\phi$ is a mediator particle. It is so special because nearly $90 \%$ of the energy from annihilation goes to the electron-positron pairs (only less than 1\% of the energy from annihilation contributes to gamma-ray). It is the reason why this channel can escape the bounds set by gamma-ray observations [6]. The ranges of dark matter mass and crosssection that can account for the positron excess are $m_{\chi}=0.59 \pm 0.02 \mathrm{TeV}$ and $<\sigma v>=$ $(5.87 \pm 0.36) \times 10^{-24} \mathrm{~cm}^{3} \mathrm{~s}^{-1}$ respectively [20] (based on the benchmark set of cosmic ray propagation model). The positron (or electron) spectrum $d N_{e} / d E$ for this channel has been computed in [22] (see Fig. 1). The high-energy positrons produced would diffuse outward and cool down within the hot gas in galaxy clusters. The equilibrium positron spectrum can be calculated by using the diffusion equation [23]:

$$
\frac{\partial}{\partial t} \frac{d n_{e}}{d E}=\nabla\left[D(E, r) \nabla \frac{d n_{e}}{d E}\right]+\frac{\partial}{\partial E}\left[b(E) \frac{d n_{e}}{d E}\right]+Q(E, r),
$$

where $d n_{e} / d E$ is the equilibrium electron/positron density spectrum, $D(E, r)$ is the spatial diffusion coefficient, $b(E)$ is the cooling rate and $Q(E, r)$ is the source term. Since the electron/positron radiation timescale is much shorter than the spatial diffusion timescale, we can neglect the time depedence term on the left hand side and the spatial dependence term on the right hand side of Eq. (1). Therefore, the equilibrium density spectrum is [23]:

$$
\frac{d n_{e}}{d E}=\frac{<\sigma v>\rho_{\chi}^{2}}{2 m_{\chi}^{2} b(E)} \int_{E}^{m_{\chi}} d E^{\prime} \frac{d N_{e}}{d E^{\prime}}
$$

where $\rho_{\chi}$ is the mass density of dark matter.

For electrons or positrons, there are four major ways of cooling: synchrotron radiation, inverse Compton scattering, Coulomb loss and bremsstrahlung [24]. Since most of the electrons or positrons produced from the $\mathrm{TeV}$ dark matter annihilation through $4-\mu$ channel have energy above $1 \mathrm{GeV}$, the cooling rate would be dominated by synchrotron and inverse Compton sccattering. Therefore, we have [23]

$$
b(E) \approx\left[0.079\left(\frac{E}{1 \mathrm{GeV}}\right)^{2}\left(\frac{B}{1 \mu \mathrm{G}}\right)^{2}+0.79\left(\frac{E}{1 \mathrm{GeV}}\right)^{2}\right] \mathrm{eV} / \mathrm{yr}
$$

where $B \sim 1 \mu \mathrm{G}$ is the magnetic field in a typical galaxy cluster [23]. 


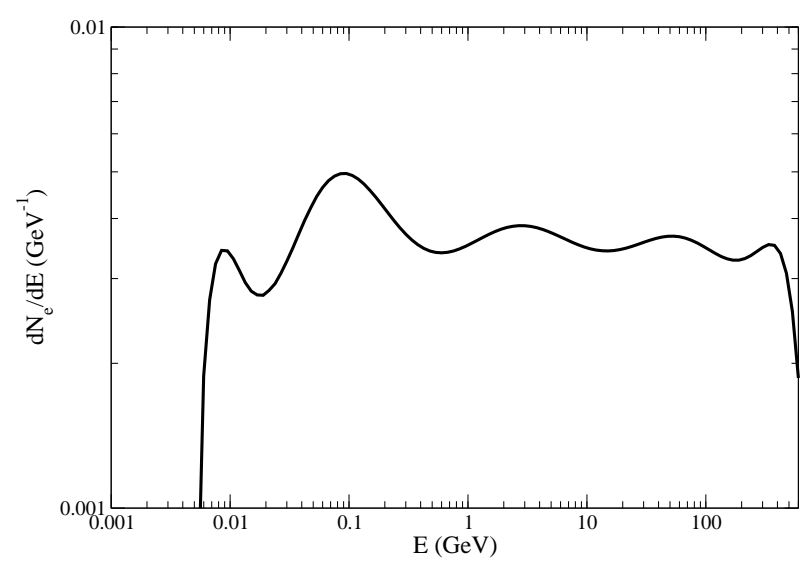

FIG. 1: The energy spectrum of positron/electron produced per one annihilation through $4-\mu$ channel [22]. Here, we use $m_{\chi}=0.59 \mathrm{TeV}$.

In equilibrium, the energy emitted by synchrotron radiation and inverse Compton scattering from electrons and positrons will finally leave the galaxy cluster and contribute to the total luminosity. The total luminosity due to the cooling of positrons and electrons within a radius $r$ in the cluster centre is

$$
L=2 \int_{0}^{r} \int_{0}^{\infty} b(E) \frac{d n_{e}}{d E} d E\left(4 \pi r^{2}\right) d r .
$$

Assume that the density of dark matter is modelled by NFW profile $\rho_{\chi}=\rho_{s} r_{s} / r$ for $r \ll r_{s}$, where $\rho_{s}$ and $r_{s}$ are scale density and scale radius respectively [25]. The values of $\rho_{s}$ and $r_{s}$ can be calculated by the mass-concentration relation [26] and the virial radius obtained from x-ray observations [28]. Therefore, by using Eq. (2), Eq. (4) can be written explicitly as

$$
L=4 \pi \frac{<\sigma v>}{m_{\chi}^{2}} \rho_{s}^{2} r_{s}^{2} r \int_{0}^{\infty} \int_{E}^{m_{\chi}} d E^{\prime} \frac{d N_{e}}{d E^{\prime}} d E .
$$

By using the energy spectrum in Fig. 1 and $m_{\chi}=0.59 \pm 0.02 \mathrm{TeV}$, the integral in the above equation is equal to $556 \mathrm{GeV}$.

\section{Compare with the observations}

In general, the luminosity due to positron and electron cooling is not very high $\left(L \sim 10^{39}\right.$

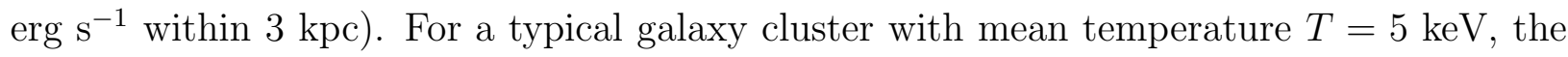
luminosity of hot gas is about $L \sim 10^{40} \mathrm{erg} \mathrm{s}^{-1}$ within $3 \mathrm{kpc}$. Nevertheless, observations reveal that some cool-cored clusters have low temperature $(T \sim 1 \mathrm{keV})$ at the centre due 
to the strong cooling signatures [30]. Therefore, the luminosity in the central part of coolcored clusters is generally lower than that of a typical galaxy cluster. Provided that the luminosity due to positron and electron cooling should not exceed the observed luminosity, these cool-cored clusters can be good candidates to constrain the parameters of dark matter annihilation.

By using the observational data from [30], we can identify four good candidates (A262, A2199, A85 and NGC5044) to achieve our purpose. These clusters are chosen since they are characterized by a temperature of the hot gas that is less than $3 \mathrm{keV}$ for $r<3 \mathrm{kpc}$. Since the observed hot gas luminosity is assumed to be dominated by bremsstrahlung radiation (the radiation from recombination contributes less than $2 \%$ of the luminosity), the observed luminosity is given by

$$
L_{o}=\Lambda_{0} T^{1 / 2} \int_{0}^{r} 4 \pi r^{2} n^{2} d r
$$

where $\Lambda_{0}=1.4 \times 10^{-27} \mathrm{erg} \mathrm{cm}^{3} \mathrm{~s}^{-1}$ and $n$ is the number density of hot gas. In general, the surface brightness profile of hot gas can be fitted with a single- $\beta$ model [27, 28]

$$
S(r)=S_{0}\left(1+\frac{r^{2}}{r_{c}^{2}}\right)^{-3 \beta+1 / 2}
$$

where $S_{0}$ is central brightness, $r_{c}$ and $\beta$ are fitted parameters. This allows us to construct the radial gas number density distribution [29]:

$$
n=n_{0}\left(1+\frac{r^{2}}{r_{c}^{2}}\right)^{-3 \beta / 2},
$$

where $n_{0}$ is the central number density. In fact, the surface brightness profile of A85 is better described by a double- $\beta$ model [28]. Nevertheless, the calculation of Eq. (6) for A85 by using the double- $\beta$ model would just give a result with about $5 \%$ smaller than that using the single- $\beta$ model. Therefore, for simplicity, we still use the single- $\beta$ model to calculate $L_{o}$. The observed temperature and the parameters used are summarized in Table 1.

By using $m_{\chi}=0.59 \pm 0.02 \mathrm{TeV}$ and $\langle\sigma v\rangle=(5.87 \pm 0.36) \times 10^{-24} \mathrm{~cm}^{3} \mathrm{~s}^{-1}$, we can calculate the lower limit of $L$ by using Eq. (5). On the other hand, by considering the uncertainties of the parameters (in Table 1), we can get the upper limit of $L_{o}$ by using Eq. (6). In Table 2, we summarize the limits of $L$ and $L_{o}$ for A262, A2199, A85 and NGC5044. We can notice that $L>L_{o}$ in A262 and A2199 while $L<L_{o}$ in A85 and NGC5044. This suggests that the cooling rate due to positrons and electrons in A262 
TABLE I: The parameters used in our calculations [28]. Here, $T$ is the temperature of the cool core in each cluster [30].

\begin{tabular}{lcccc}
\hline & $\mathrm{A} 262$ & $\mathrm{~A} 2199$ & $\mathrm{~A} 85$ & NGC5044 \\
\hline$\rho_{s}\left(10^{14} M_{\odot} \mathrm{Mpc}^{-3}\right)$ & 14.1 & 9.56 & 8.34 & 14.7 \\
$r_{s}(\mathrm{kpc})$ & 172 & 334 & 444 & 127 \\
$\beta$ & $0.443_{-0.017}^{+0.018}$ & $0.665_{-0.021}^{+0.019}$ & $0.532_{-0.004}^{+0.004}$ & $0.524_{-0.003}^{+0.002}$ \\
$r_{c}(\mathrm{kpc})$ & $41_{-9}^{+11}$ & $139_{-9}^{+10}$ & $82_{-3}^{+3}$ & $11_{-0}^{+0}$ \\
$n_{0}\left(10^{-2} \mathrm{~cm}^{-3}\right)$ & $0.81_{-0.09}^{+0.13}$ & $0.83_{-0.03}^{+0.03}$ & $2.57_{-0.10}^{+0.10}$ & $3.45_{-0.03}^{+0.03}$ \\
$T(\mathrm{keV})$ & $<1($ for $r<2 \mathrm{kpc})<2($ for $r<3 \mathrm{kpc})<3($ for $r<3 \mathrm{kpc})<0.8($ for $r<1 \mathrm{kpc})$ \\
\hline
\end{tabular}

TABLE II: The luminosity due to positron/electron cooling $L$ and the observed luminosity of the cool core $L_{o}$. Here, we use $m_{\chi}=(0.59 \pm 0.02) \mathrm{TeV}$ and $\left\langle\sigma v>=(5.87 \pm 0.36) \times 10^{-24} \mathrm{~cm}^{3} \mathrm{~s}^{-1}\right.$.

\begin{tabular}{lcc}
\hline Cluster & $L\left(\mathrm{erg} \mathrm{s}^{-1}\right)$ & $L_{o}\left(\mathrm{erg} \mathrm{s}^{-1}\right)$ \\
\hline A262 & $>8.3 \times 10^{38}$ & $<4.2 \times 10^{38}$ \\
A2199 & $>2.2 \times 10^{39}$ & $<1.7 \times 10^{39}$ \\
A85 & $>2.9 \times 10^{39}$ & $<2.0 \times 10^{40}$ \\
NGC5044 & $>2.5 \times 10^{38}$ & $<6.4 \times 10^{38}$ \\
\hline
\end{tabular}

and A2199 is too high. The temperature should be much less than the observed one. To avoid exceeding the observed luminosity, the upper limit of the annihilation cross-section constrained by A262 is $<\sigma v><2 \times 10^{-24} \mathrm{~cm}^{3} \mathrm{~s}^{-1}$ for $m_{\chi}=0.59 \mathrm{TeV}$, which is a few times smaller than the proposed one that can account for the excess positrons. In other words, the $4-\mu$ annihilation channel should be ruled out unless the dark matter cross-section and rest mass are significantly deviated from the best-fit values.

\section{Discussion}

In this article, we show that the cooling of the positrons and electrons produced from dark matter annihilation through $4-\mu$ channel can produce a significant amount of energy. This energy is a few times larger than the observed luminosity in two cool-cored galaxy clusters (A262 and A2199). It suggests that a dark matter annihilation through the $4-\mu$ 
channel with a cross-section sufficiently large to fit the high-energy positrons excess is not possible.

However, the $4-\mu$ channel is the only viable leptophilic channel to reconcile the tension between dark matter interpretation of excess positrons seen in AMS-02 and the gamma-ray constraint from dwarf galaxies. If our analysis is correct, all the leptophilic channels are ruled out by observations. Nevertheless, in the above calculations, we just have 2 galaxy clusters to perform the analysis. More observational data from cool-cored clusters can provide a better verification in this issue.

Nevertheless, it is also possible to have other annihilation channels, such as quarks, vector and Higgs boson channels. The required dark matter mass is heavier than $\sim 10 \mathrm{TeV}$, which would produce antiprotons at high energy. Recent analysis from [31] suggests that dark matter annihilation through $b \bar{b}$ or $W^{+} W^{-}$can provide good fits to the cosmic antiproton to proton ratio up to $\sim 450 \mathrm{GeV}$. For example, for $W^{+} W^{-}$channel, the required ranges of mass and cross-section of dark matter are $m_{\chi}=(4.4-36.3) \mathrm{TeV}$ and $\langle\sigma v\rangle=\left(3.7 \times 10^{-24}-\right.$ $\left.3.5 \times 10^{-22}\right) \mathrm{cm}^{3} \mathrm{~s}^{-1}$ respectively [31]. Obviously, the ranges are too wide to constrain the properties of dark matter. Moreover, the required cross-section is smaller than the one that can account for the excess positrons seen in AMS-02 $\left.(<\sigma v\rangle=(5.10 \pm 0.48) \times 10^{-22} \mathrm{~cm}^{3} \mathrm{~s}^{-1}\right)$ [20]. The same problem also applies to $b \bar{b}$ channel. Therefore, there is some inconsistency between the results from antiproton data and positron data. By combining all the results and constraints, it leaves only a small window for dark matter interpretation to resolve the conflicts among the positron data (AMS-02, HEAT, PAMELA), antiproton data (AMS-02), and gamma-ray data (Fermi/LAT).

[1] J. J. Beatty et al., Phys. Rev. Lett., 93, 241102 (2014).

[2] O. Adriani et al., Phys. Rev. Lett., 111, 081102 (2013).

[3] M. Aguilar et al., Phys. Rev. Lett., 110, 141102 (2013).

[4] L. Accardo et al., Phys. Rev. Lett., 113, 121101 (2014).

[5] M. Aguilar et al., Phys. Rev. Lett., 113, 121102 (2014).

[6] A. Lopez, C. Savage, D. Spolyar and D. Q. Adams, arXiv:1501.01618.

[7] D. Hooper, P. Blasi and P. D. Serpico, JCAP 0901, 025 (2009) 
[8] H. Yuksel, M. D. Kistler and T. Stanev, Phys. Rev. Lett., 103, 051101 (2009).

[9] T. Delahaye, J. Lavalle, R. Lineros, F. Donato and N. Fornengo, Astron. Astrophys., 524, A51 (2010).

[10] S. Profumo, Central Eur. J. Phys., 10, 1 (2011).

[11] T. Linden and S. Profumo, Astrophys. J., 772, 18 (2013).

[12] T. Delahaye, K. Kotera and J. Silk, Astrophys. J., 794, 168 (2014).

[13] M. Di Mauro, F. Donato, N. Fornengo, R. Lineros and A. Vittino, JCAP, 1404, 006 (2014).

[14] T. Delahaye, R. Lineros, F. Donato, N. Fornengo and P. Salati, Phys. Rev. D, 77, 063527 (2008).

[15] M. Cirelli, M. Kadastik, M. Raidal and A. Strumia, Nucl. Phys. B, 813, 1 (2009).

[16] L. Bergstrom, T. Bringmann, I. Cholis, D. Hooper and C. Weniger, Phys. Rev. Lett., 111, $171101(2013)$.

[17] V. C. Spanos, arXiv:1312.7841.

[18] A. Ibarra, A. S. Lamperstorfer and J. Silk, Phys. Rev. D, 89, 063539 (2014).

[19] Q.-H. Cao, C.-R. Chen and T. Gong, arXiv:1409.7317.

[20] M. Boudaud et al., Astron. Astrophys., 575, A67 (2015).

[21] A. Sommerfeld, Annalen der Physik, 403, 257 (1931).

[22] M. Cirelli, et al., JCAP, 1210, E01 (2012).

[23] E. Storm, T. E. Jeltema, S. Profumo and L. Rudnick, Astrophys. J., 768, 106 (2013).

[24] M. S. Longair, High Energy Astrophysics, vol. 2 (Cambridge: Cambridge University Press, 1994).

[25] J. F. Navarro, C. S. Frenk and S. D. M. White, ApJ, 490, 493 (1997).

[26] M. Schaller, et al., MNRAS, 451, 1247 (2015).

[27] A. Cavaliere and R. Fusco-Femiano, Astron. Astrophys., 49, 137 (1976).

[28] Y. Chen, T. H. Reiprich, H. Böhringer, Y. Ikebe and Y.-Y. Zhang, Astron. Astrophys., 466, 805 (2007).

[29] T. H. Reiprich and H. Böhringer, Astrophys. J., 567, 716 (2002).

[30] A. J. R. Sanderson, T. J. Ponman and E. O’Sullivan, MNRAS, 372, 1496 (2006).

[31] S.-J. Lin, X.-J. Bi, P.-F. Yin and Z.-H. Yu, arXiv:1504.07230. 